accelerated atherosclerosis. However, studies on the prevalence and impact on the peripheral vascular beds remain scarce.

The aim of this study was to determine the prevalence of vascular affection in SLE patients, defined as an altered anklebrachial index (ABI), carotid intima-media thickness (IMT), doppler-duplex interrogation and clinical evaluation by a vascular surgeon, as well as its relationship to clinical and serological variables.

Methods Eighty consecutive SLE patients ( $\geq 4$ ACR criteria) from an inception cohort were studied. Each patient had a vascular clinical evaluation and was screened for ankle-brachial index (ABI) and carotid intima-media thickness (IMT) after 12.1 (3.8) yrs of follow-up. In patients with a history of deep vein thrombosis (DVT), a doppler-duplex ultrasound interrogation was added to the evaluation.

Results The mean age of the patients was 39 years (SD 9). Of the 80 patients studied, $4 \%$ had an abnormal ABI, $8.7 \%$ had a history of DVT, and $15 \%$ had an altered carotid IMT. The global prevalence of any-kind vascular disease was $21.2 \%$. Variables strongly correlated to vascular disease were current age, age at diagnosis and $\operatorname{IgM}$ anticardiolipin (aCL $\operatorname{IgM}$ ) antibody. Conclusions The increased prevalence of a diverse range of vascular diseases in this young population of patients with SLE justifies a vascular evaluation in every patient to identify, follow and potentially treat them in a timely fashion.

\section{D-DIMER AS AN EARLY MARKER IN PATIENTS WITH LUPUS MESENTERIC VASCULITIS}

X Ma*, X Feng, H Wang, B Hua, L Sun. Drum Tower Hospital - Medical School of Nanjing University, Rheumatology, Nanjing, China

\subsection{6/lupus-2017-000215.175}

Background and aims No early serum marker contributes to the diagnosis of lupus mesenteric vasculitis (LMV). The aim of this study was to investigate clinical significance of serum d-dimer level as an early diagnosis marker of LMV patients.

Methods The 57 systemic lupus erythematosus (SLE) patients were retrospectively analysed and classified into LMV group $(n=19)$ and Non-LMV group $(n=38)$ between May 2010 and January 2016. The serum d-dimer level was measured on the first day after SLE patients presented acute abdomen as well as imaging, other laboratory-testing parameters, and SLEDAI during the same period. The maximum and mean d-dimer values were analysed and compared with other potential markers for diagnosis of LMV. The correlation of d-dimer level with other potential severity markers and inflammation parameters were also studied.

Results Both maximum and mean d-dimer level on the first day of presentation of acute abdomen were significantly higher in LMV patients. The d-dimer level was correlated well with 1-lactate and SLEDAI. In addition, d-dimer level was detected poor correlation with white blood cell count and Creactive protein level.

Conclusions d-dimer level could be an effective and early serum diagnosis marker of LMV.

\section{ACCRUAL OF DISEASE COMORBIDITIES OVER 8 YEARS IN A MULTICENTRE INCEPTION SLE COHORT}

${ }^{1} \mathrm{M}$ Urowitz, ${ }^{1} \mathrm{D}$ Gladman*, ${ }^{2} \mathrm{~J} \mathrm{Su},{ }^{2} \mathrm{~N}$ Anderson. Systemic Lupus International Collaborating Clnics; 'University of Toronto, Medicine, Toronto, Canada; ${ }^{2}$ Toronto Western Hospital, Rheumatology, Toronto, Canada

\subsection{6/lupus-2017-000215.176}

Background and aims The annual accrual of comorbidities in patients with SLE is not well described. We report the annual occurrence of these features in an inception cohort of patients with SLE.

Methods An international research network comprised of 33 centres from 11 countries has followed an inception cohort of SLE patients 2yearly according to a standardised protocol between 2000 and 2016. Of these, 717 patients followed for a minimum of 8 years constitute the study population. Comorbidities including atherosclerotic vascular events (AVEs), osteoporosis, osteonecrosis and diabetes are assessed using the SLICC/ACR Damage Index (SLICC/DI). AVEs are described and attributed on a specialised form. Diagnosis of an event is confirmed using standard clinical criteria, relevant laboratory data and imaging where appropriate. Descriptive statistics were used.

Abstract 176 Table 1 Cumulative SDI, AVEs, steoporosis, osteonecrosis and diabetes in the firest 8 years of follow-up ( $n=17)$.

\begin{tabular}{|l|c|c|c|c|c|}
\hline $\begin{array}{c}\text { Follow- } \\
\text { up }\end{array}$ & $\begin{array}{c}\text { SDI } \\
\text { (meantstd) }\end{array}$ & AVE & Osteoporosis & Osteonecrosis & Diabetes \\
\hline 1 & $0.3 \pm 0.7$ & $4(0.5 \%)$ & $3(0.4)$ & $3(0.4 \%)$ & $13(1.8 \%)$ \\
\hline 2 & $0.5 \pm 1.0$ & $5(0.7 \%)$ & $5(0.7 \%)$ & $10(1.4 \%)$ & $17(2.4 \%)$ \\
\hline 3 & $0.6 \pm 1.2$ & $10(1.4 \%)$ & $7(1.0 \%)$ & $15(2.1 \%)$ & $17(2.4 \%)$ \\
\hline 4 & $0.7 \pm 1.2$ & $13(1.8 \%)$ & $10(1.4 \%)$ & $18(2.5 \%)$ & $19(2.7 \%)$ \\
\hline 5 & $0.8 \pm 1.3$ & $18(2.5 \%)$ & $11(1.5 \%)$ & $21(2.9 \%)$ & $19(2.7 \%)$ \\
\hline 6 & $1.0 \pm 1.4$ & $20(2.8 \%)$ & $15(2.1 \%)$ & $25(3.5 \%)$ & $21(2.9 \%)$ \\
\hline 7 & $1.0 \pm 1.4$ & $21(2.9 \%)$ & $17(2.4 \%)$ & $27(3.8 \%)$ & $22(3.1 \%)$ \\
\hline 8 & $1.1 \pm 1.5$ & $25(3.5 \%)$ & $19(2.7 \%)$ & $31(4.3 \%)$ & $25(3.5 \%)$ \\
\hline
\end{tabular}


Abstract 176 Table 2 Cumulative comorbidities by year in caucasians compared to all other ethnicities

\begin{tabular}{|l|c|c|c|c|c|c|c|c|}
\hline & \multicolumn{7}{|c|}{ Follow up years since SLE diagnosis } \\
\hline Comorbidity & 1 & 2 & 3 & 4 & 5 & 6 & 7 & 8 \\
\hline AVE Caucasian (\%) & 0.59 & 0.88 & 2.06 & 2.95 & 3.54 & 4.13 & 4.42 & 5.60 \\
\hline AVE Other (\%) & 0.53 & 0.53 & 0.79 & 0.79 & 1.59 & 1.59 & 1.59 & 1.59 \\
\hline Osteoporosis Caucasians (\%) & 0.00 & 0.59 & 1.18 & 1.77 & 2.06 & 3.25 & 3.55 & 4.14 \\
\hline Osteoporosis Others (\%) & 0.79 & 0.79 & 0.79 & 1.06 & 1.06 & 1.06 & 1.32 & 1.32 \\
\hline Osteonecrosis Caucasians (\%) & 0.59 & 1.18 & 1.18 & 1.47 & 1.47 & 2.07 & 2.07 & 2.66 \\
\hline Osteonecrosis Others (\%) & 0.26 & 1.59 & 2.91 & 3.44 & 4.23 & 4.76 & 5.29 & 5.82 \\
\hline Diabetes Caucasian (\%) & 1.77 & 2.65 & 2.65 & 2.95 & 2.95 & 2.96 & 2.96 & 3.25 \\
\hline Diabetes Other (\%) & 1.85 & 2.12 & 2.12 & 2.38 & 2.38 & 2.91 & 3.17 & 3.70 \\
\hline
\end{tabular}

Results Of the 717 patients followed for at least 8 years, 90.2\% were female, $47.3 \%$ were Caucasian, $13.8 \%$ were Black, $19.4 \%$ were Asian, $16.3 \%$ Hispanic and 3.2\% other. Their mean age at enrolment was $34.2 \pm 13.1$ years and SLEDAI- $2 \mathrm{~K}$ at enrolment was $4.17 \pm 4.49$. The duration from diagnosis to enrolment was $5.9 \pm 4.4$ months.

Mean SDI gradually increases over 8 years. The accumulation of AVEs, osteoporosis, osteonecrosis and diabetes all increase progressively over an 8 year period. Caucasians accumulate AVEs and osteoporosis more frequently than all "other" ethnicities. In contrast, all "other" ethnicities accumulate osteonecrosis more frequently than Caucasians. All ethnicities accumulate diabetes at the same frequency.

Conclusions As expected disease damage and comorbidities in newly diagnosed patients increase over their first 8 years. Different ethnicities accumulate comorbidities at different rates.

\section{ASSOCIATION BETWEEN CHRONIC ANTIMALARIAL THERAPY AND ELEVATED MYOCARDIAL BIOMARKERS IN PATIENTS WITH SYSTEMIC LUPUS ERYTHEMATOSUS AND NO PRIOR HEART DISEASE: A PRELIMINARY REPORT}

${ }^{1} \mathrm{~K}$ Tselios, ${ }^{1} \mathrm{D}$ Gladman*, ${ }^{2} \mathrm{P}$ Harvey, ${ }^{1} \mathrm{~S} \mathrm{Su},{ }^{1} \mathrm{M}$ Urowitz. ${ }^{1}$ University of Toronto and Toronto Western Hospital, Rheumatology, Toronto, Canada; ${ }^{2}$ University of Toronto and Women's College Hospital, Medicine, Toronto, Canada

\subsection{6/lupus-2017-000215.177}

Background and aims Antimalarial (AM)-induced cardiomyopathy is an extremely rare complication of AM treatment in systemic lupus erythematosus (SLE). The use of specific cardiac biomarkers may identify patients at risk. We sought to investigate the prevalence and associated factors for abnormal myocardial biomarkers in lupus patients.

Methods Consecutive patients $(n=179)$ attending the Toronto Lupus Clinic were enrolled. BNP (brain natriuretic peptide, assessing pressure and/or volume overload) and cTnI (cardiac troponin I, assessing myocardial necrosis) were measured simultaneously. None had ECG abnormalities suggestive of acute coronary syndrome. Analysis was performed with SAS $9.3 ; \mathrm{p}<0.05$ was considered significant.
Results Twenty-seven patients (15.1\%) had elevated BNP and/ or cTnI; 11 with prior history of heart failure, coronary artery disease, pulmonary hypertension and/or exertional dyspnea were excluded. Compared to subjects with normal biomarkers, the remaining patients $(n=16)$ were older [54.7 \pm 15.1 vs. $47.8 \pm 12.2$ years, $p=0.037]$, had longer disease duration [22.6 \pm 10.4 vs. $15.5 \pm 10.1$ years, $\mathrm{p}<0.001]$, longer $\mathrm{AM}$ use $[12.5 \pm 9.6$ vs. $7.9 \pm 8$ years, $\mathrm{p}=0.034]$ and more frequently persistent CPK elevation [44.4 vs. $16.4 \%, \mathrm{p}<0.001]$. Multi-variable regression analysis showed chronic AM treatment combined with CPK elevation to be an important predictor for elevated myocardial biomarkers $[\mathrm{HR}=1.41$, 95\% $\mathrm{CI}=1.06-1.89, \mathrm{p}=0.02]$. Two patients were diagnosed with AM-induced cardiomyopathy on endomyocardial biopsy; both had CPK and BNP/cTnI elevation.

Conclusions Approximately 9\% of unselected SLE patients had elevated myocardial biomarkers, in the absence of prior cardiac disease. Chronic AM therapy accompanied by persistent CPK elevation conferred an increased risk for abnormal BNP and cTnI, which might predict cardiomyopathy in such patients.

\section{ASSESSMENT OF THE RISK OF FLARES IN SYSTEMIC LUPUS ERITHEMATOSUS}

${ }^{1} \mathrm{~L}$ Mazur-Nicorici*, ${ }^{1} \mathrm{~V}$ Sadovici-Bobeica, ${ }^{1} \mathrm{M}$ Garabajiu, ${ }^{2} \mathrm{~N}$ Revenco, ${ }^{1} \mathrm{M}$ Mazur. ${ }^{1}$ State Medical and Pharmaceutical University Nicolae Testemitanu, Department of Internal Medicine, Chisinau, Moldova; ${ }^{2}$ State Medical and Pharmaceutical University Nicolae Testemitanu, Department of Pediatry, Chisinau, Moldova

\subsection{6/lupus-2017-000215.178}

Background and aims A prospective analytic study was conducted during February 2015-February 2016, which included adult patients with SLE, according to SLICC, 2012 classification criteria. The subjects were prospectively fallowed-up every month 3, 5, 9, 12 visit and disease activity by SLEDAI and SLAM, SELENA/SLEDAI flare index and laboratory tests were assessed. The risk of flare was calculated by Pearson correlation coefficient and risk ratio (RR) with 95\% CI.

Results In the study were included 102 SLE patients, 94,1\% females, mean age \pm SD $42.4 \pm 13.3$ (range 20-73) years, mean disease duration \pm SD93.9 \pm 77.1 (range 0,1-228) months. 